\title{
Factores de riesgo y características de la gestación en la adolescente y su hijo
}

\author{
Angela Escobar*; Néstor Balcázar**
}

\begin{abstract}
RESUMEN: Los riesgos biológicos, sociales y psicológicos de la gestación en adolescentes aumentan la morbilidad y mortalidad maternofetal.

Prospectivamente se tomaron 161 adolescentes embarazadas atendidas en el Hospital Infantil Lorencita Villegas de Santos, las cuales se dividieron en dos grupos de acuerdo a si tuvieron control prenatal en la institución o no; y, por otra parte y para efectos del estudio, según su edad, en menores de dieciséis años y mayores de diecisiete años. Se describen las características demográficas del embarazo, del parto y del recién nacido para cada grupo, y se mencionan por aparte las diferencias que se observaron en algunos tópicos. Por ejemplo, la asociación entre hipertensión inducida por el embarazo y adolescencia fue más alta en nuestras pacientes que la reportada por la literatura. Así mismo, fue mayor entre las adolescentes no controladas por este servicio. En cuanto al tipo de parto, el índice de cesáreas es el mismo que el de las pacientes adultas en esta institución, sinembargo la indicación para las adolescentes en el $76 \%$ fue la desproporción cefalopélvica de causa materna.
\end{abstract}

Con la adecuación de la dieta a las pacientes controladas por nuestro servicio, obtuvimos una menor tasa de anemia durante el embarazo $(p=0,005)$ y un porcentaje menor y estadísticamente significativo de bajo peso al nacer de los recién nacidos; lo cual sugiere que es de vital importancia un adecuado control prenatal específico y multidisciplinario para adolescentes.

PALABRAS CLAVES: Embarazo, Adolescentes, Factores de Riesgo.

SUMMARY: The biologic, social and psychologic risks augment the fetus-maternal morbility and mortality in pregnant adolescents.

Prospectively, they were taken 161 pregnant adolescents attended in the "Hospital Lorencita Villegas de Santos". They were divided into two groups according whether if they had prenatal control or not; and, by another side and for effects of the study, according to the age, they were divided into youngers than sixteen years and into olders than sixteen years. There are described the demographic characteristics of pregnancy, of childbirth and of the recently born child for each group, and there are mentioned the differences observed in some topics. For example, the association between hypertension induced by pregnancy and adolescence was higher in our patients than the reported ones by literature. Equally, it was greater between the non-controled adolescents by this service. Reffering to the type of childhood, the index of caesarean operations is the same as the one of adult patients in this institution, although the indication for the adolescents in the $76 \%$ was the cephalu-pelvic desproportion of matern cause.

With the adecuation of a diet to the patients controled by our service, we obtained a minor rate of anemia during pregnancy $(P=0,005)$ and a lower percentage and statisticly meaningly of low weight in the birth of the recently borned; what suggests that it is of vital importance a prenatal specific and multidisciplinary control for adolescents.

KEY WORD: Pregnancy, Adolescents, Risk factors.

\section{Introducción}

Los riesgos biológicos, sociales, psicológicos de la gestación en adolescentes aumentan la morbilidad y mortalidad materno-fetal (1-2).

Durante los últimos quince años, la opinión pública se ha formulado en políticas de investigación relacionadas con la morbilidad y mortalidad en la sexualidad, embarazos y crianzas de los hijos de adolescentes; esfuerzo éste que ha resultado cargado de matices políticos, morales y religiosos que han hecho que se dificulte el

Residente III año. Departamento de Ginecología y Obstetricia. Hospital Infantil Universitario Lorencita Villegas de Santos. Instructor. Departamento de Ginecología y Obstetricia a cargo de la consulta de adolescentes. Hospital Infantil Universitario Lorencita Villegas de Santos. análisis objetivo de esta entidad, que hoy por hoy toma carácter de problema de salud pública de primer orden.

Debe entenderse por adolescente embarazada la mujer que se encuentra en estado de gravidez, en edades comprendidas entre los diecinueve años según la O.M.S. Edades entre las que se encuentra mayor morbimortalidad para la madre y su hijo, debido a factores de índole biológico, cultural, psicológico, económico y personal (1, 3).

La adolescencia es un período de la vida de comienzo y duración variable, que marca el final de la niñez y crea los cimientos de la maduración y la vida adulta. Su desarrollo en los niveles biológicos, psicológico y social, está marcado por una interacción significativa, de modo que los acontecimientos o alteraciones en cualquiera de estos niveles puede retrasar o acelerar la evolución de cada uno de los demás (4). 
Desde el punto de vista biológico, su inicio está señalado por la aceleración final del crecimiento y la aparición del desarrollo sexual secundario; su terminación está marcada por la fusión de las epífisis y la finalización de la diferenciación sexual.

Como característica fundamental está la pubertad, que es la época en que empieza a manifestarse la actitud para la reproducción. Vale decir, el tiempo en que se desarrollan los caracteres sexuales secundarios, se presenta la menarquia, y se efectúan los cambios psicosexuales. Es el inicio de la maduración sexual y física (5-6). Los cambios observados varían de una niña a otra tanto en tiempo como en intensidad y el estudio de estos parámetros se relaciona entre sí. Ellos son: Ganancia de peso y talla, desarrollo mamario, aparición y crecimiento del vello púbico, axilar y por último, la menarquia $(5,7-8)$.

El cambio psicológico de la adolescente se muestra por una aceleración del desarrollo cognitivo y la formación de la personalidad, que va seguida de la asunción del papel parental. Lo más llamativo de la adolescencia es laconsecución de la capacidad de conceptualización abstracta, que evoluciona los cimientos de la creatividad, la contribución científica y que también explica la preocupación creciente por los problemas sociales y políticos, por los significados y valores básicos de la existencia humana (9-11). Este idealismo conduce a interrogar y examinar las premisas fundamentales y a una insatisfacción con las imperfecciones del mundo tal como es. En la búsqueda de un sentimiento de identidad personal, el adolescente que ya no es un niño ni todavía un adulto, se dedica afanosamente a determinar quién es y en quién debe convertirse (12).

Socialmente la adolescencia es un período de preparación intensificada para la adopción de un papel adulto, y su terminación queda sancionada cuando se otorga al adolescente prerrogativas adultas plenas, cuya naturaleza y momento de concesión varían ampliamente de una sociedad a otra. $(10,13)$. La adolescencia como fenómeno social, aun cuando sea limitada por términos biológicos, es función de normas culturales. Mientras más complicada sea la tecnología de una sociedad, más prolongado es el período de la adolescencia, puesto que la complejidad de la preparación necesaria para los papeles adultos depende de las exigencias de la sociedad (14).

Por otra parte el desarrollo de las características sexuales adultas y la experiencia de un conjunto desconcertante de nuevas sensaciones físicas, ponen a prueba las estructuras psicosexuales de la infancia. El papel de las hormonas se limita a dejar a punto el organismo para la maduración biológica, a influir pero no determinar el nivel de la líbido. La dirección, naturaleza y adecuación de las realizaciones sexuales son controladas por factores psicosociales.

Dentro del grupo de adolescentes, podemos identificar tres grupos:

Temprano: Que se inicia alrededor de los diez a los trece años, que corresponde al inicio mismo de la pubertad y finaliza a los catorce o quince años. Este grupo presenta un alto riesgo respecto al embarazo, ya que puede ser sexualmente activo, ser presa fácil de adultos $o$ adolescentes mayores.

Medio: Que continúa a la anterior y finaliza alrededor de los diecisiete años.

Tardío: Que termina a los diecinueve años cumplidos para unos y a los veintiuno para otros (15-16).

Para efectos de este marco teórico, la adolescencia va de los diez años a los diecinueve años cumplidos.

Respecto al embarazo la edad puede clasificarse de manera diferente,siendo pacientes menores de dieciséis años el primer grupo, consideradas como de mayor riesgo, y las pacientes mayores de dieciséis años, con riesgo menor a las anteriores, pero mayor con relación a las de veinte a treinta años $(5,17-19)$.

Los aspectos que rodean las causas del embarazo en adolescentes están inmersos en un contexto socio-cultural que cambia con rapidez: Por ejemplo, debido en parte a la revolución sexual que se dio en los años sesentasetenta, las adolescentes tienen una proporción creciente de actividad sexual; de tal suerte que en el decenio de los ochenta, alrededor de los doce años la actividad sexual es del 2 al $3 \%$ y va aumentando de acuerdo a la edad: $8 \%$ a los catorce años, $25 \%$ a los dieciséis años y finalmente entre el 50-59\% a los diecinueve años. (7, 10, 20-21). Se estima que una de cada diez adolescentes queda embarazada, dando lugar al 10 -

$13 \%$ de los nacidos vivos en Colombia, y a un porcentaje desconocido de los abortos provocados en el país ( 9 , 22-23).

Por otro lado, la actividad sexual temprana suele relacionarse con menarquias cada vez más tempranas, que conducen a un desarrollo corporal más temprano y a concentraciones hormonales incrementadas en estas adolescentes, que junto a profundos cambios en la moral sexual, a cambios socio-culturales y educacionales, hipertrofia juvenil, independencia de la mujer, los medios de comunicación y el desconocimiento o mal uso de los métodos de planificación familiar, dan origen a los preocupantes porcentajes mencionados $(11,24-25)$.

Estas particularidades han hecho que los estudiosos describan a la adolescente como Riesgo de Embarazo con algunas características que incluyen:

- Bajo rendimiento escolar o indisciplina.

- Hijas de madres cabeza-de-hogar.

- Hijas de madres que a su vez fueron madres-adolescentes.

- Niñas abandonadas por sus madres.

- Adolescentes que adoptan conductas de riesgo y rebeldía.

- Malas relaciones con sus padres $(7,14,26)$.

El embarazo y la tenencia de hijos durante la adolescencia entrañan un riesgo médico más elevado para la madre y el niño, lo mismo que desventajas económicas, sociales y académicas duraderas para los padres adolescentes y su hijo. Se ha observado por ejemplo, que los hijos de madres adolescentes tienen mayor riesgo de presentar déficitis cognoscitivos y psicológicos; como defectos manifiestos de una maternidadde mala calidad, 
estado socioeconómico más bajo y expectativas de futuro desventajosas $(3,11)$.

Las madres adolescentes, principalmente de quince años y menos, tienen mayor peligro de presentar desproporción feto-pélvica, anemia, toxemia e infecciones genitales y urinarias. De igual forma, tienden a tener menos controles prenatales, a tenerlos tardíos o a no tenerlos, quizá porque no se percatan o no admiten estar embarazadas y también porque no pueden o no quieren tener asistencia médica (27).

En términos de riesgos clínicos para la madre adolescente, el más grave es la muerte por complicaciones gestacionales en niñas que tienen menos de quince años, el cual puede ser de cuatro veces más elevado con relación a mujeres embarazadas de veinte a treinta años $(3,7,18)$.

En Colombia se ha encontrado una incidencia de toxemia en adolescentes de $9 \%$ al $19 \%$, eclampsia de $1.66 \%$, interrupciones precoces del embarazo del 7 al $10 \%$; índice de prematuridad del 16 al $23 \%$, e incluso en un estudio del $48 \%$ y un aumento importante en las malformaciones congénitas en menores de catorce años $(5,12,15,28)$.

En los datos encontrados de la morbi-mortalidad maternofetal, se encuentra además: Aumentos en el tiempo de trabajo de parto, mayor incidencia de partos pretérmino, elevada mortalidad perinatal y bajo peso al nacer, el cual se ha estimado como del 18 al $26 \%$ en menores de dieciséis años, y del 9 al $13 \%$ en menores de diecinueve años $(8,13,19)$. Este bajo peso al nacer tiene un origen multifactorial, pero está íntimamente relacionado con un inadecuado control prenatal, amén de los aspectos socioculturales ya mencionados.

Finalmente, se ha observado un aumento en la mortalidad infantil en los primeros dos años de vida, relacionada con la probabilidad más alta de abuso y maltrato por parte de sus padres, y mayor frecuencia en accidentes como quemaduras, envenenamientos y traumatismos. De otro lado, estos niños tienen mayores problemas de aprendizaje, comportamiento y desarrollo (14, 16, 2930).

Por todas estas razones se han diseñado y puesto en marcha varios centros de atención integral de adolescentes en Estados Unidos, Europa y algunos países Suramericanos, lo mismo que en el Hospital Infantil Universitario "Lorencita Villegas de Santos" de Bogotá, con programas prenatales y perinatales que pretenden mejorar los resultados de los malos embarazos, disminuir los niveles de tensión en las madres y sus familias, recurriendo a planes de asistencia de casos individuales y prevención denuevos embarazos, dando como resultado la ocurrencia de menos complicaciones del nacimiento, menos morbi-mortalidad perinatal y un número reducido de lactantes de bajo peso al nacer. Estos programas también enseñan a las madres adolescentes como resolver sus problemas encontrando su bienestar psicológico, mejorando su rendimiento cognitivo y sus niveles de apoyo social.

En el departamento de Ginecobstetricia del Hospital Infantil Lorencita Villegas de Santos, no hay un trabajo descriptivo con el grupo de adolescentes, que describa los factores de riesgo, las características de la gestación en estas madres, la morbimortalidad y los resultados perinatales, que permita además conocer específicamente la problemática de las pacientes que acuden a nuestra institución, y pueda servir de partida para otros trabajos prospectivos con adolescentes embarazadas.

Asimismo, se quiere aprovechar la oportunidad para consolidar y desarrollar mejor aún el manejo integral e interdisciplinario que se tiene en la actualidad en el Departamento de Ginecología y Obstetricia del Hospital con este grupo tan especial de pacientes, el cual comprende las siguientes partes:

Valoración y asistencia por trabajo social

Control prenatal por la consulta de adolescentes

Control prenatal por la unidad de medicina materno fetal ( $\mathrm{Si}$ es pertinente)

Valoración y asesoría por psicología de adolescentes

Valoración y asesoría por nutrición

Curso de adecuación para la maternidad de adolescentes

Y como objetivos tenemos el reconocimiento de la situación actual del grupo de adolescentes embarazadas y sus recién nacidos en el Hospital Infantil Lorencita Villegas de Santos, la determinación de la prevalencia de factores de riesgo ya conocidos entre un grupo de pacientes controladas y otro de no controladas, y además la descripción de morbimortalidad perinatal en estos grupos.

\section{Material y métodos}

El estudio se realizó del 1-V-91 al 30-IV-92, tomando como población a todas las Adolescentes Embarazadas (10 a 19 años cumplidos) que asistieron al Hospital Infantil Lorencita Villegas de Santos, tanto a los controles de la consulta de Adolescentes, como las que consultaron al Servicio para la atención de su parto y no tuvieron control prenatal en el Hospital. De tal manera se dividió a la población en dos grupos: Grupo I-Pacientes con control en el Hospital y, Grupo II- Pacientes sin control prenatal en el Hospital. Además, para efectos prácticos y con base en observaciones propias y ajenas, se decidió dividir el grupo en pacientes de 16 años y menores, y pacientes entre los 17 y los 19 años, las cuales parecen tener un comportamiento similar entre sí.

A las pacientes que tuvieron control en el Hospital se les tomó una historia clínica completa, aplicando el mismo formato que se tiene en el Departamento de Ginecología y Obstetricia. Dentro de las variables tenemos datos de la edad, estado civil, antecedentes familiares, antecedentes personales y patología asociada al embarazo, antecedentes gineco-obstétricos. Como datos adicionales se interrogó sobre la escolaridad, deseo o no del embarazo y el peso anterior al mismo. Para las pacientes no controladas en el Hospital, dicha información se tomó de la historia clínica realizada en admisiones, en el momento del ingreso. Respecto a los datos del parto y la valoración inicial del recién nacido, se tomaron de la historia obstétrica y pediátrica de cada adolescente lo referente a: Peso, Talla, Apgar y Sexo. 
Con el objetivo de establecer si había relación entre los eventos de tener control prenatal o no y los resultados de Morbimortalidad materna y fetal, se determinó la frecuencia de algunas complicaciones perinatales como Amenaza de Trabajo de Parto Prematuro, Hipertensión Inducida por el Embarazo, Anemia, Infección de Vías Urinarias, Endometritis, Sufrimiento Fetal Agudo, Apgar menor de 5.

Una vez completado el tiempo del estudio y revisado las historias clínicas, se procedió a la tabulación de los datos para su análisis estadístico, el cual se hizo con las pruebas de chi cuadrado y Exacta de Fischer, y dado que se hicieron múltiples comparaciones, se consideró significativo un valor de $\mathrm{p}<\mathrm{o}=\mathrm{a} 0.005$.

\section{Resultados}

Durante el año comprendido entre el 1-V-91 y el 30-IV92 se atendieron en el Hospital Infantil Universitario Lorencita Villegas de Santos, un total de 2.597 partos, de los cuales $161(6.2 \%)$ fueron de menores de 19 años cumplidos, porcentaje inferior al observado en otras ocasiones en la misma institución que fue en promedio del $11 \%$. De estas 161 pacientes, el $26 \%(n=42)$ fueron menores de 16 años.

MUESTRA LOS RESULTADOS DEMOGRAFICOS Y GENERALES OBTENIDOS:

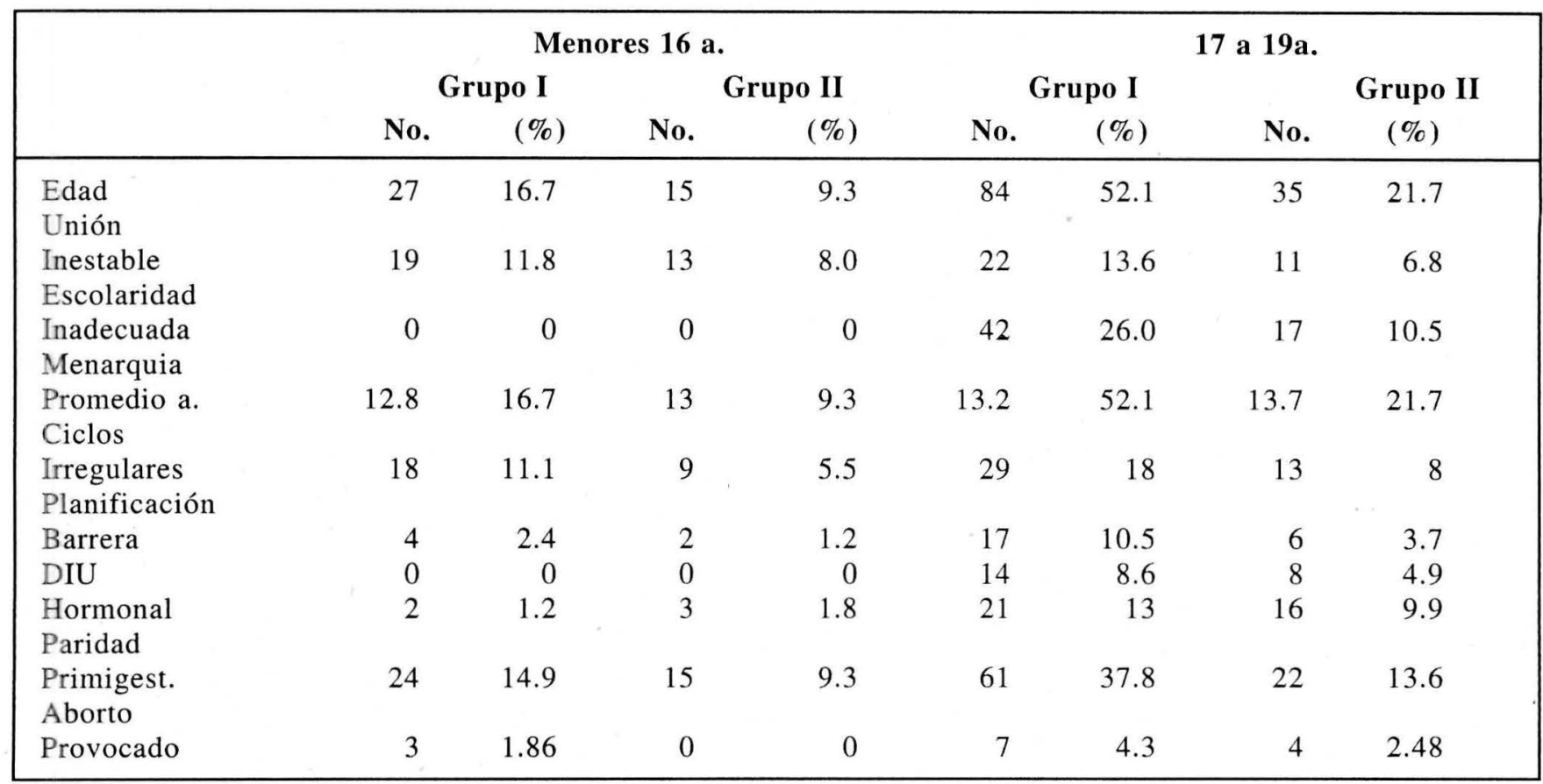

Tabla 1-2

CARACTERISTICAS DEL EMBARAZO ACTUAL

\begin{tabular}{|c|c|c|c|c|c|c|c|c|}
\hline & \multicolumn{4}{|c|}{ Menores de 16 a. } & \multicolumn{4}{|c|}{17 a 19 años } \\
\hline & \multicolumn{2}{|c|}{ Grupo I } & \multicolumn{2}{|c|}{ Grupo II } & \multicolumn{2}{|c|}{ Grupo I } & \multicolumn{2}{|c|}{ Grupo II } \\
\hline & No. & $(\%)$ & No. & $(\%)$ & No. & $(\%)$ & No. & $(\%)$ \\
\hline \multicolumn{9}{|l|}{ Embarazo } \\
\hline No deseo & 21 & 13 & 13 & 8 & 69 & 42.8 & 11 & 6.8 \\
\hline 1a. Cita & & & & & & & & \\
\hline Semanas & 30.2 & 16.7 & 39.2 & 9.3 & 28 & 52.1 & 36.4 & 21.7 \\
\hline \multicolumn{9}{|l|}{ Complic. } \\
\hline Madre: & & & & & & & & \\
\hline Anemia & 2 & 1.2 & 3 & 1.8 & 6 & 3.7 & 9 & 5.5 \\
\hline Toxemia & 4 & 2.4 & 8 & 4.9 & 19 & 11.8 & 12 & 7.4 \\
\hline I.V.U. & 8 & 4.9 & 4 & 2.4 & 19 & 11.8 & 9 & 5.5 \\
\hline Endometr. & 1 & 0.6 & 3 & 1.8 & 6 & 3.7 & 6 & 3.7 \\
\hline
\end{tabular}


Tabla 1-3

CARACTERISTICAS Y COMPLICACIONES

DEL PARTO

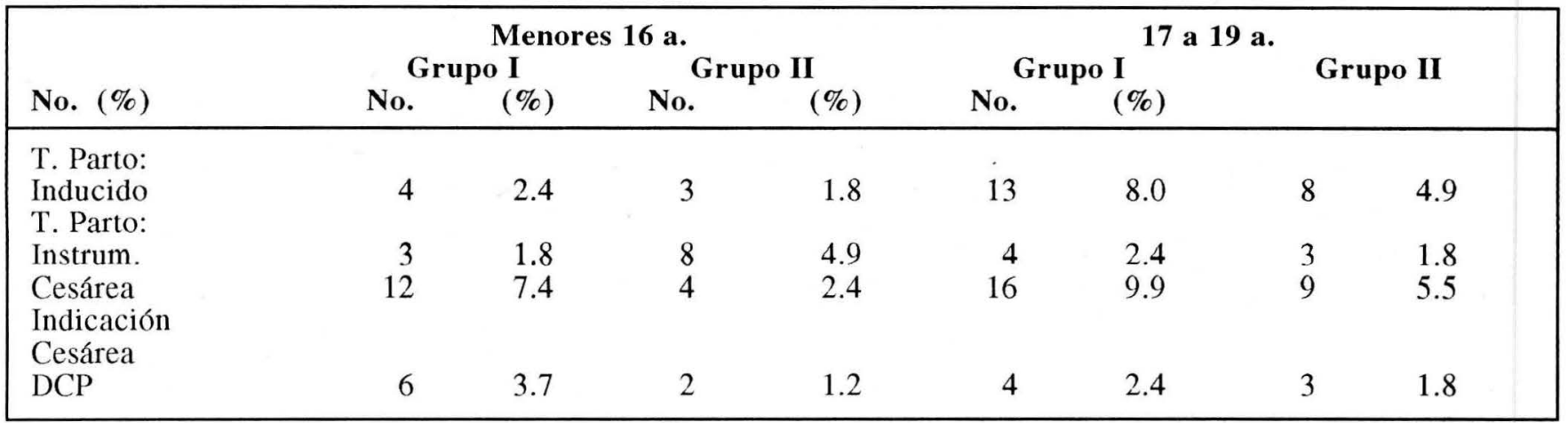

Tabla 1-4

CARACTERISTICAS DEL RECIEN NACIDO

\begin{tabular}{|c|c|c|c|c|c|c|c|c|}
\hline & \multicolumn{4}{|c|}{ Menores 16 a. } & \multicolumn{4}{|c|}{17 a 19 a. } \\
\hline & \multicolumn{2}{|c|}{ Grupo I } & \multicolumn{2}{|c|}{ Grupo II } & \multicolumn{2}{|c|}{ Grupo I } & \multicolumn{2}{|c|}{ Grupo II } \\
\hline & No. & $(\%)$ & No. & $(\%)$ & No. & $(\%)$ & No. & $(\%)$ \\
\hline $\begin{array}{l}\text { Bajo peso } \\
\text { al nacer }\end{array}$ & 4 & 2.4 & 6 & 3.7 & 11 & 6.8 & 15 & 9.3 \\
\hline Apgar $<5$ & 2 & 1.2 & 2 & 1.2 & 7 & 4.3 & 6 & 3.7 \\
\hline S.F.A. & 5 & 3.1 & 5 & 3.1 & 13 & 8.0 & 12 & 7.4 \\
\hline
\end{tabular}

El uso de los métodos de planificación hormonales en el grupo de adolescentes fue dado exclusivamente para las mayores; en cambio métodos como los de barrera son utilizados por las menores de 16a. en un $11.8 \%$.

Dentro de las patologías asociadas al embarazo como la toxemia se tuvo una incidencia del 14 y $32 \%$ del total de las adolescentes, siendo el porcentaje más alto en las pacientes no controladas. (fig. 1 y 2 ). La anemia se presentó en el 3-12\% de las adolescentes, con un porcentaje más alto estadísticamente significativo en las meno-

Figura 1

HIPERTENSION INDUCIDA POR EL EMBARAZO (MENORES 16 AÑOS)

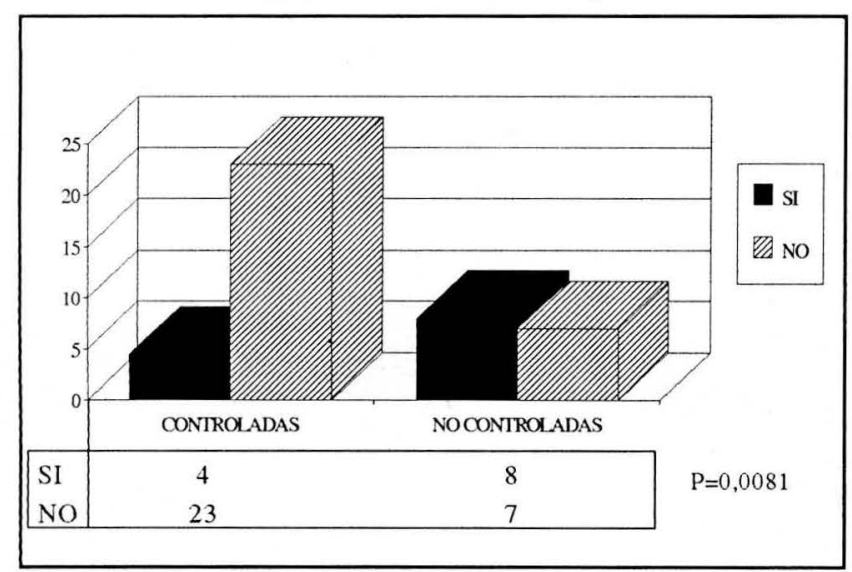

res de 16a. no controladas por este servicio. (fig. 3 y 4). La diferencia en la frecuencia de parto pretérmino, no es estadísticamente significativa entre los dos grupos estudiados. De otra parte, sí se observa diferencia entre los grupos respecto al diagnóstico de sufrimiento fetal agudo (fig. 5 y 6 ).

El índice de partos intervenidos por cesárea fue en promedio para las menores del $25 \%$ y para las mayores del $26 \%$, en la mayoría su única indicación fue la desproporción feto-pélvica.

\section{Figura 2 \\ HIPERTENSION INDUCIDA POR EL EMBARAZO (17 A 19 AÑOS)}

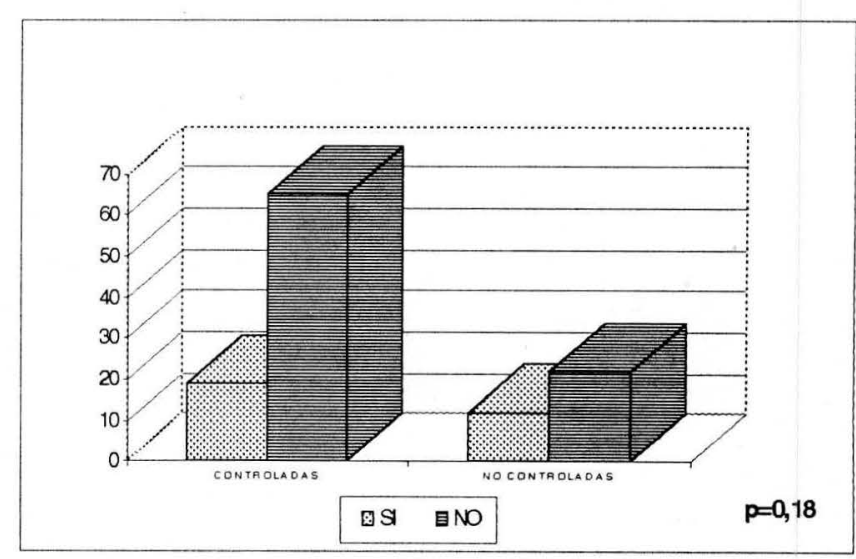


Figura 3

SUFRIMIENTO FETAL AGUDO

(MENORES DE 16 AÑOS)

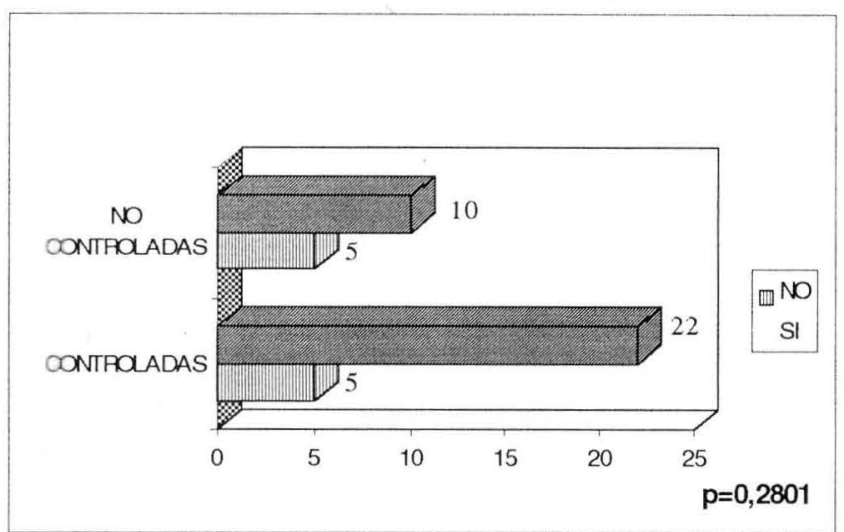

Figura 5

APGAR $<5$ (MENORES DE 16 AÑOS)

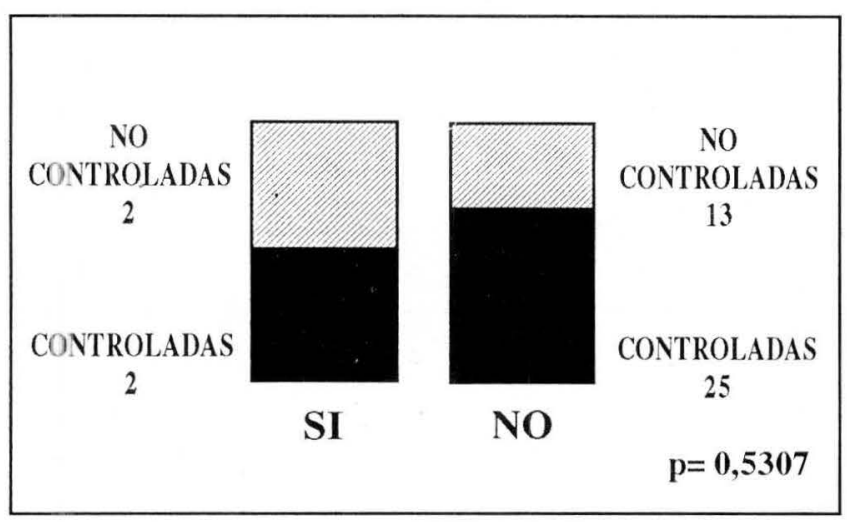

En los resultados perinatales se vio a la valoración del apgar $<5$, que el grupo de mayores no controladas fue el más elevado porcentaje (fig. 7 y 8). El porcentaje de bajo peso al nacer es mucho menor estadísticamente significativo en el grupo de pacientes adolescentes controladas

Figura 7

ANEMIA (MENORES DE 16 AÑOS)

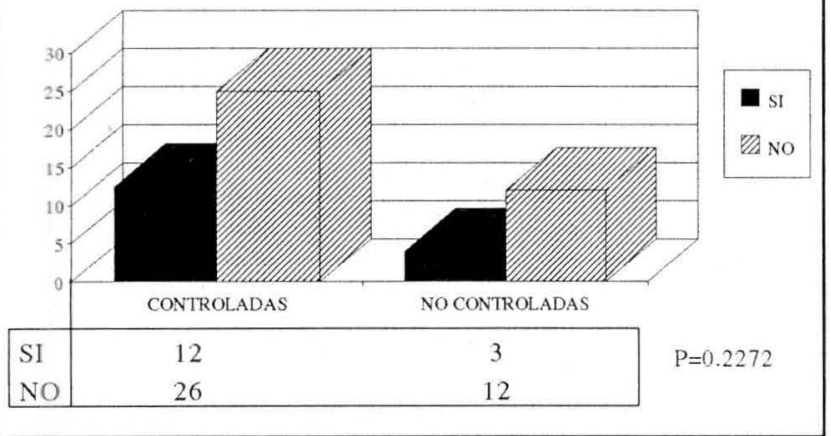

Figura 4

SUFRIMIENTO FETAL AGUDO

(17 A 19 A ÑOS)

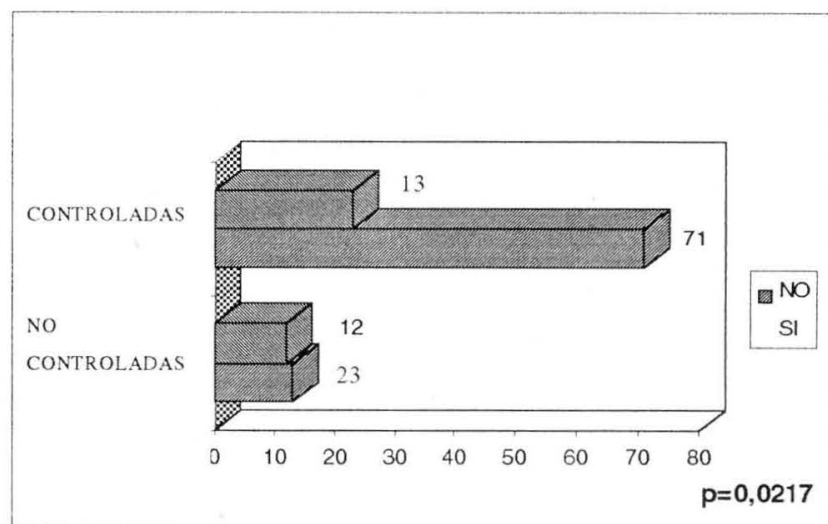

Figura 6

APGAR $<5(17$ A 19 A ÑOS $)$

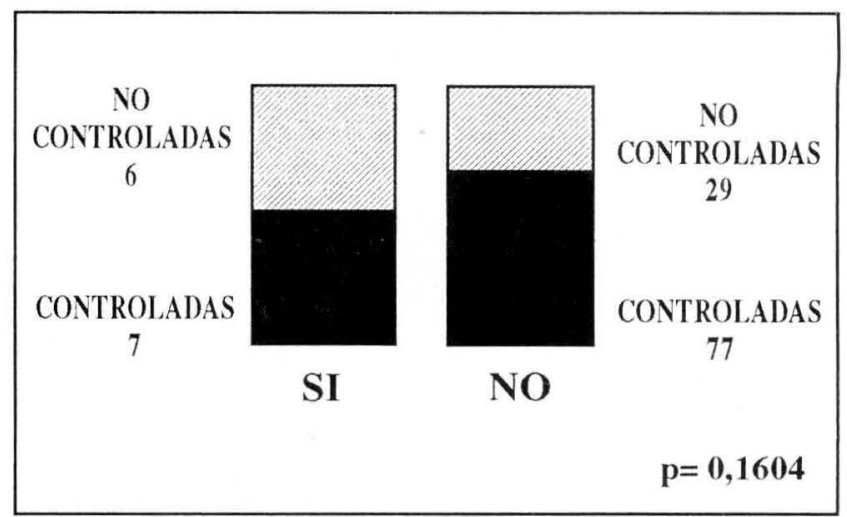

por nosotros, que recibieron toda la asesoría nutricional correspondiente.

Y para culminar comparando un poco la morbilidad materna que también es importante, se tomó el parámetro de endometritis postparto (fig. 9 y 10).

Figura 8

ANEMIA (17 A 19 A N NOS

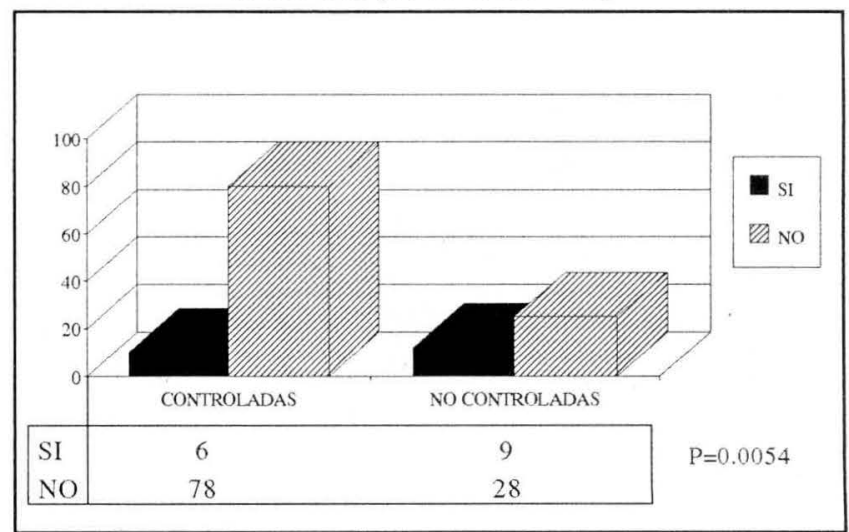


Figura 9

ENDOMETRITIS (MENORES DE 16 AÑOS)

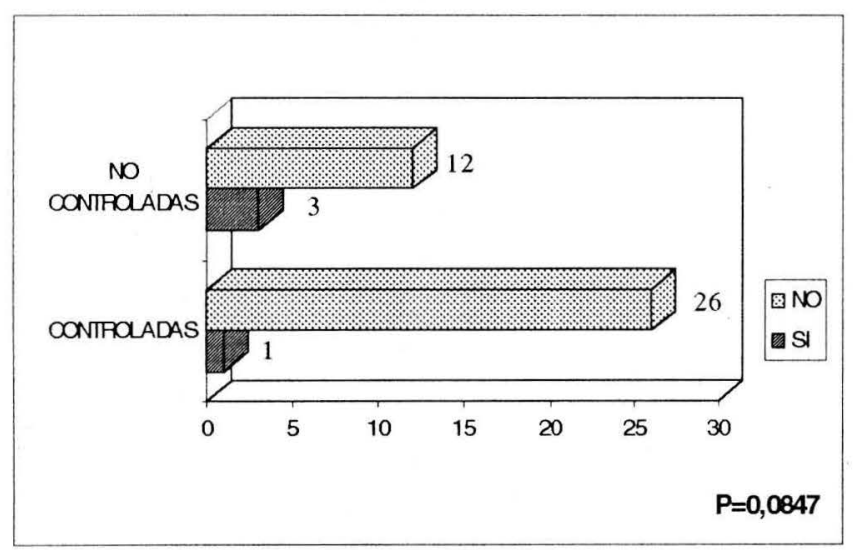

\section{Discusión}

Los resultados sugieren que un inadecuado cuidado prenatal y una historia obstétrica adversa son importantes factores de riesgo para desarrollar anemia dentro del embarazo así como de obtener recién nacidos de más bajo peso al nacer. Dentro de las variables que se tuvieron en cuenta importantes, que seguramente influyeron en los resultados, son la adecuación nutricional en la dieta de estas madres adolescentescontroladas por el servicio; anotando que el número de controles prenatales y el tiempo al cual asistieron en su primera visita, es de vital importancia en estos resultados.

Aunque los hallazgos no fueron estadísticamente significativos de asociación entre hipertensión inducida por el embarazo y edad, se pudo observar que tenemos un índice de toxemia más alto que el reportado en otras instituciones y la literatura $(23,25)$; siendo del $20.7 \%$ en adolescentes controladas y del $40 \%$ en las no controladas; predominando el grupo de los 17-19 años. Explicándonos estos hallazgos tal vez por el tamaño de la muestra, que estamos en un hospital de remisión, o debido a que se puede estar sobrediagnosticando la toxemia.

Patologías como la infección urinaria, curiosamente se presentó en mayor número para las pacientes controladas que las no controladas; muy seguramente por pasar inadvertidas. El tipo de parto especialmente la cesárea, el porcentaje no es mayor a lo que se describe con respecto a las adultas; lo que si es de tener en cuenta, su indicación en casi todas las cesáreas practicadas a adolescentes, fue la desproporción feto-pélvica.

La endometritis post-parto fue un $10 \%$ mayor en promedio, para las adolescentes no controladas que para
Figura 10

ENDOMETRITIS (17 A 19 AÑOS)

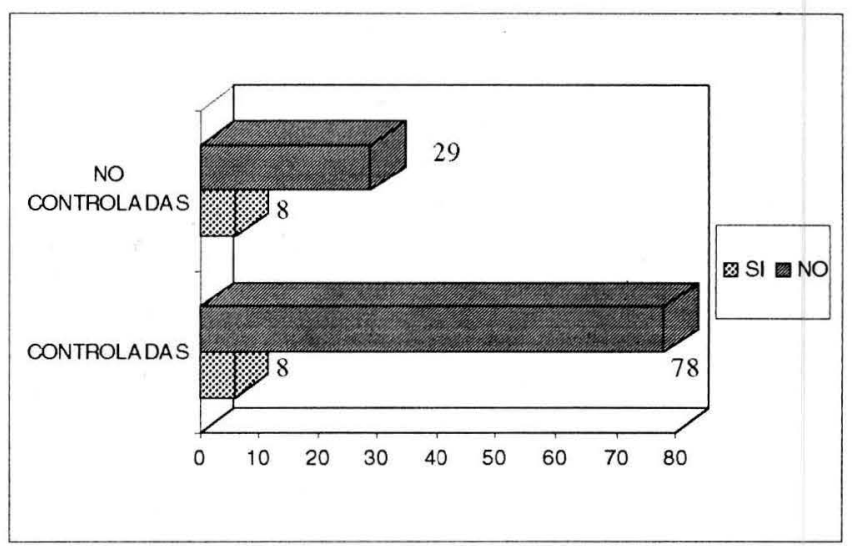

las controladas en el hospital y así mismo aumentada especialmente en el grupo de adolescentes entre 17-19 años.

Los hallazgos se correlacionan con lo descrito en las diferentes revisiones bibliográficas de la mayoría de artículos que hablan de factores de riesgo en los embarazos de adolescentes, aunque de todas formas es un trabajo descriptivo donde se observaron asociaciones y frecuencias, nos permite conocer mejor nuestra población hospitalaria de madres adolescentes.

\section{Conclusiones}

- La adecuación nutricional mejora el peso del recién nacido producto de un embarazo de madre adolescente.

- El aporte de hierro en tercer trimestre disminuye la anemia en estas adolescentes.

\section{Recomendaciones}

- Realizar un trabajo prospectivo específicamente sobre toxemia en adolescentes controladas en el hospital.

- Promover campañas de información sobre sexualidad, anticoncepción y embarazo en los colegios, tratando de incentivar la consulta temprana dela niña al ginecólogo y de la embarazada al obstetra.

- Mejorar y tener en cuenta los parámetros de valoración de la pelvis en las adolescentes, con el fin de que toda adolescente de por sí, no sea causa del diagnóstico de desproporción feto-pélvica y termine en un parto por cesárea.

\section{BIBLIOGRAFIA}

1. Newcomer SF., Udry JR. Adolescent sexual behavior and popularity. Adolescence 1983; 18: 515-517.

2. National Research Council: Panel on adolescent pregnancy and childbearing. Risking the future: Adolescent sexuality, pregnancy and childbearing. National Academy Press. 1987; 1 : $39-47$.
3. Freedman MA., Kaplan JH Cols. Compendio de Psiquiatría. Barcelona: Salvat, $1984 ; 638$.

4. Martín E. Problemática sexual del adolescente (Tesis). Bogotá: Hospital Infantil Lorencita Villegas de Santos. 1986; 36.

5. Wallace H., Vienonen M. Teenage pregnancy in Sweden and Finland. J. Adolesc Health Care 1989; 10: 231-236. 
6. Miller PY., Simon W. Adolescent sexual behavior: Context and change. Soc. Probl. 1974; 22: 58-76.

7. Mcanarney ER., Hendee WR. Adolescent pregnancy and its consequences. JAMA 1989; 262: 74-77.

8. Matsuhashi Y., Felice $\mathrm{M}$ et al. Is repeat pregnancy in adolescents a "planned" affair? J. Adolesc Health Care 1989; 10: 409-412.

9. Duarte Contreras A. Ginecología de la niña y la adolescente. Barcelona: Salvat, 1988; 443.

10. Mcanarney ER., Hendee WR et al. The prevention of adolescent pregnancy. JAMA 1989; 262: 78-81.

11. Jekel JF., Tyler NC et al. Induced abortion and sterilization amongwomen who became mothers as adolescents. Am. J. Public. Health 1977; 67: 621-625.

12. Zabin SL., Hirsch BM et al. Differential Characteristics of adolescent pregnancy test patients: Abortion, childbearing and negative test groups. J. Adolesc. Health Care 1990; 11: 107-113.

13. Moreno RA. Adolescentes y embarazo. Rev. Col. Osbtet. y Ginecol. 1989; 40: 227-230.

14. Jones EF., Forrest JD et al. Teenage pregnancy in developed countries Determinants and policy implications. Fam. Plann. Perspect. 1985 17: 53-63.

15. Díaz A., Jaffe LR et al. Frecuency of use, knowledge, and attitudes toward the contraceptive sponge among inner-city black and Hispanic adolescent females. J. Adolesc. Health Care 1990; 11: 125-127.

16. Hofferth SL., Kahn JR et al. Premarital sexual activity among US teenage women over the past three decades. Fam Plann Perspect 1985; 17: 53-63.

17. Card J., Wise L. Teenage mothers and teenage fathers: The impact of early childbearing on the parent's personal and professional lives. Fam Plann Perspect 1978; 10: 199-205.

18. Elkind D. Egocentrims in adolescence. Child Develop 1967; 38 1025-1034.
19. Duarte CA., Barreto LA. Factores determinantes del embarazo en adolescentes solteras. Rev. Col. Ginecol. y Obstet. 1985; 36: 291295.

20. Juahasz AM., Sonnensheim SM. Adolescent decision-making: Components and skills. Adolescence 1980; 15: 743-746.

21. Jessor SL., Jessor R. Transition from virginity to nonvirginity among youth: A social psychological study over time. Dev. Psychol 1975; 11: $473-475$

22. Graham D. The obstetric and neonatal consequences of adolescent pregnancy. Birth Defects 1981; 17: 49-67.

23. Gómez PJ. Embarazo en adolescentes. Rev. Col. Ginecol. y Obstet. 1972; 23: 353-356.

24. Makinson $\mathrm{C}$. The health consequences of teenage fertility. Fam Plann Perspect 1985; 17: 132-134.

25. Tovar MC., Burbano C y Cols. Estudio comparativo embarazo de adolescentes vs embarazo adultas jóvenes en el centro hospital primitivo iglesias de Cali. Rev. Col. Ginecol. y Obstet. 1987; 38: 436-440.

26. Slap BG., Schwartz SJ. Risk factors for low birth weight to adolescentmothers. J. Adolesc. Health care 1989; 10: 267-274.

27. Zuravin S. Child maltratment and teenage first births: A relationship mediated by chronic sociodemographic stress? Am. J. Orthopsychiatry 1988; 58: 91-97.

28. Schinke SP., Barth RP et al. Adolescent mothers, stress, and prevention. J. Human Stress 1986; 12: 162-165.

29. Lawrence S., Neinstein. Adolescent Health Care. 2nd ed. Baltimore Urban and Schwarsenberg 1991; 89.

30. Creasy RK., Resnik N. Maternal-fetal medicine: Principles and practice. W.B. Saunders Co. 1989; 784.

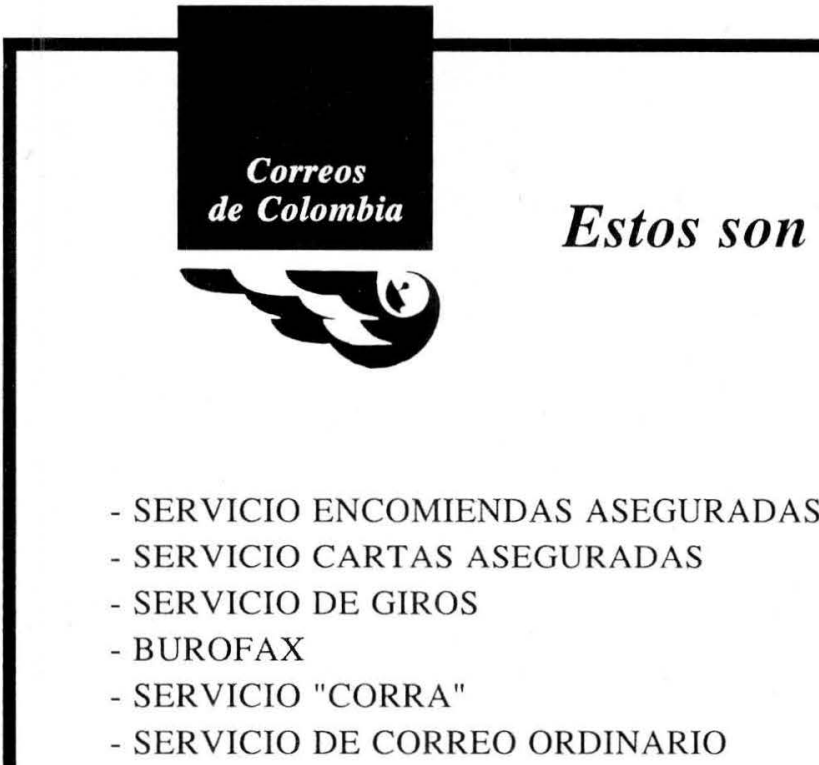

- SERVICIO DE CORREO CERTIFICADO

- SERVICIO DE CERTIFICADO ESPECIAL

- ENCOMIENDAS CONTRA REEMBOLSO

- SERVICIO TARIFA POSTAL REDUCIDA

- SERVICIO DE FILATELIA

- SERVICIO ELECTRONICO

- SERVICIO INTERNACIONAL APR/SAL

- SERVICIO RESPUESTA COMERCIAL

- SERVICIOS ESPECIALES

Teléfonos para quejas y reclamos: 3340304 - 3415536 - Santafé de Bogotá, D.C. - Colombia Cuente con nosotros hay que creer en los Correos de Colombia 